\title{
Innovative Design of Equipment and Tools. Innovative Systems with Elements of Artificial Intelligence and Neural Networks
}

\author{
Dmitrii Shekhovtsov
}

\begin{abstract}
A set of new and rather unusual conditions and requirements for all stages of design, pre-production and complex production itself, which aims to create the capabilities and specifics of so-called smart production, machines, transport, technologies and smart jobs in all industries, medicine, science, training of specialists and agriculture, make us reconsider the existing order of things and search for new organizational and technical solutions aimed at ensuring compliance with the newly emerged conditions. The author of the publication considers it important to consider this as a model within the sector of the technological field of medical equipment and its accompanying materials and technologies.
\end{abstract}

Keywords: smart production, Artificial Intelligence, medical equipment, neural networks

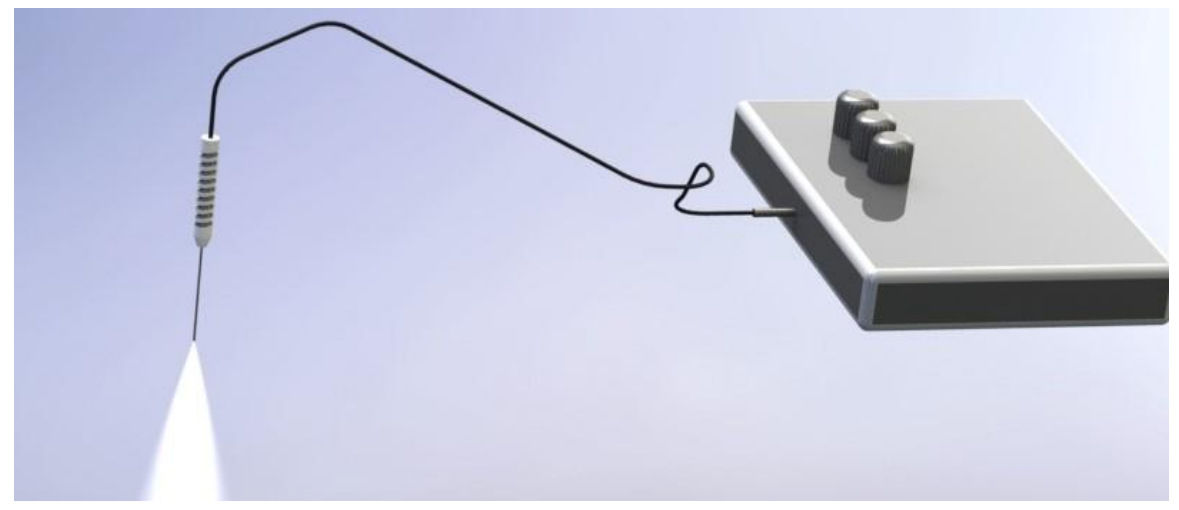

Рисунок 1. Инновационная надсистема комплексного лазерного инструмента для применения в офтальмологии

В системе используется одноразовый инструмент, связанный с источниками лазерного излучения при помощи прецизионного оптического кабеля с инструментом на одном конце и с кодирующим устройством на другом конце, причём в системе в целом применяются люминофоры , задачей которых является получения требуемого спектра излучения одновременно с смягчением влияния лазерного излучения высокой концентрации и интенсивности на физиологические параметры организма пациента.

Надо сказать, что за последнее время созданы инновационные интегративные сочетания передовых эффективных технологий , позволяющих при минимальных производственных затратах, соизмеримых с затратами на обычные носители и накопители информации, создавать надсистемы в которых носителями информации являются терабитные носители и их эквиваленты , причём системные связи носителей 
информации с элементами искусственного интеллекта и искусственными нейронными сетями в корне меняют параметры технических характеристик и технических возможностей надсистем и входящих в них иерархий структур подсистем.

Эти технологии параллельно сформированы группами разработчиков из нескольких стран, включая Японию и Южную Корею.

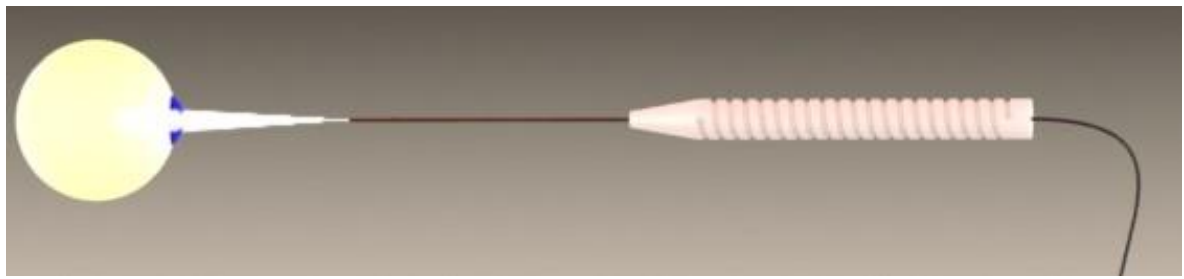

Рисунок 2. Одноразовый лазерный инструмент с схемой излучения в глазное яблоко пациента

На рисунке 2 показан одноразовый лазерный инструмент с схемой излучения в глазное яблоко пациента, у которого было выполнено несколько условий и приёмов оптимизации параметров и спектра излучения. Всё это было выполнено благодаря наличию специальных драйверов и оптических миксеров, позволяющих при использовании трёх лазерных диодов одинаковой мощности получить оптимальный вид, спектр и мощность излучения.

Как видно из рисунка, вследствие использования одноразового инструмента, возникает необходимость предельно точного кодирования для того, чтобы исключить возможность малейших ошибок при настройке и оптимизации параметров излучения, которые могут возникнуть при использовании поддельных одноразовых инструментов не имеющих требуемых параметров настройки и адаптации к условиям проведения операции.

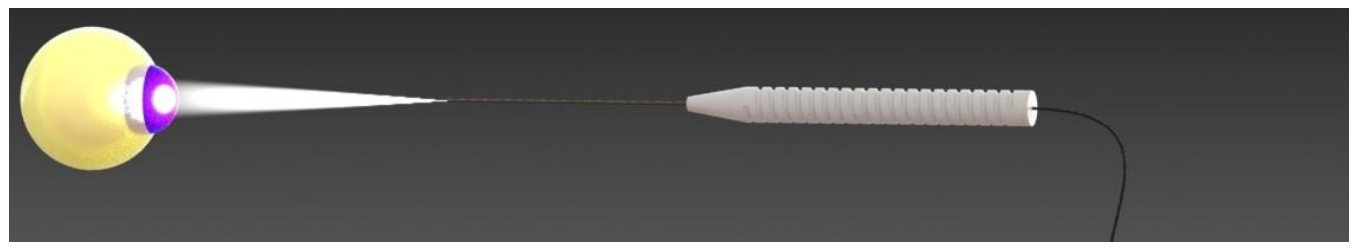

Рисунок 3. Вариант одноразового лазерного инструмента 
На рисунке показан вариант одноразового лазерного инструмента, формирующий, совместно с оптическими компонентами глазного яблока пациента районы оптической аберрации, способствующие более адаптивным включением активного интенсивного лазерного луча в сочетания оптических условий, возникших в обрабатываемом глазном яблоке пациента. Эти варианты оптической интеграции излучения одноразового инструмента инновационной надсистемы комплексного драйвера и других составляющих лазерного инструмента для применения в офтальмологии определены наличием постоянной функциональной связи между процессорами драйверов для каждого из трёх лазерных диодов и между элементами искусственного интеллекта и искусственными нейронными полями.

Показанная компоновочная гибкость во всей надсистеме позволяет при будущих модификациях рассчитывать на уверенное использование в системах управления и контроля также и квантовых компьютеров и их процессорных эквивалентов, возможно и в сочетании или в симбиозе с наиболее продвинутыми вариантами современной компьютерной и процессорной техники, в том числе и с электронными платами , произведёнными по РИТМ технологиям (техника размерного избирательного травления металла ).

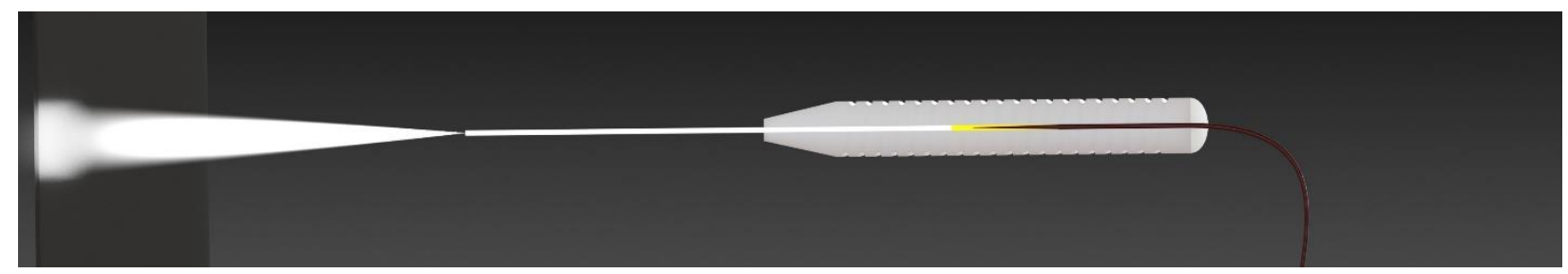

Рисунок 4. Вариант одноразового лазерного инструмента

На рисунке 4 также показан вариант одноразового лазерного инструмента, формирующий совместно с оптическими компонентами глазного яблока пациента районы оптической аберрации, способствующие более адаптивным включением активного интенсивного лазерного луча в сочетания оптических условий, возникших в обрабатываемом глазном яблоке пациента. Но в этом случае часть роли в комплексном процессе адаптации принимает на себя применение в обработке и покрытии оптического кабеля (волокна) различных видов люминофоров. Эти варианты оптической интеграции 
излучения одноразового инструмента инновационной надсистемы комплексного драйвера и других составляющих лазерного инструмента для применения в офтальмологии определены также дополнительным фактором влияния свойств и возможностей люминофора в сочетании с наличием постоянной функциональной связи между процессорами драйверов для каждого из трёх лазерных диодов и между элементами искусственного интеллекта и искусственными нейронными полями, на которые в данном случае накладывается дополнительная задача по скоростной детальной координации всех факторов формирующих конечную техническую характеристику одноразового инструмента.

Показанная компоновочная гибкость во всей надсистеме позволяет при будущих модификациях рассчитывать на уверенное использование в системах управления и контроля также и квантовых компьютеров и их процессорных эквивалентов с уже сегодня доказанным сверхвысоким быстродействием, возможно и в сочетании или в симбиозе с наиболее продвинутыми вариантами современной компьютерной и процессорной техники, в том числе и с электронными платами, произведёнными по РИТМ технологиям ( техника размерного избирательного травления металла ), которые только за счёт своей конструкции и, особенно за счёт расстояния между слоями печатной платы всего в 50 микрон, увеличивают быстродействие и скорость реакции в сотни раз.

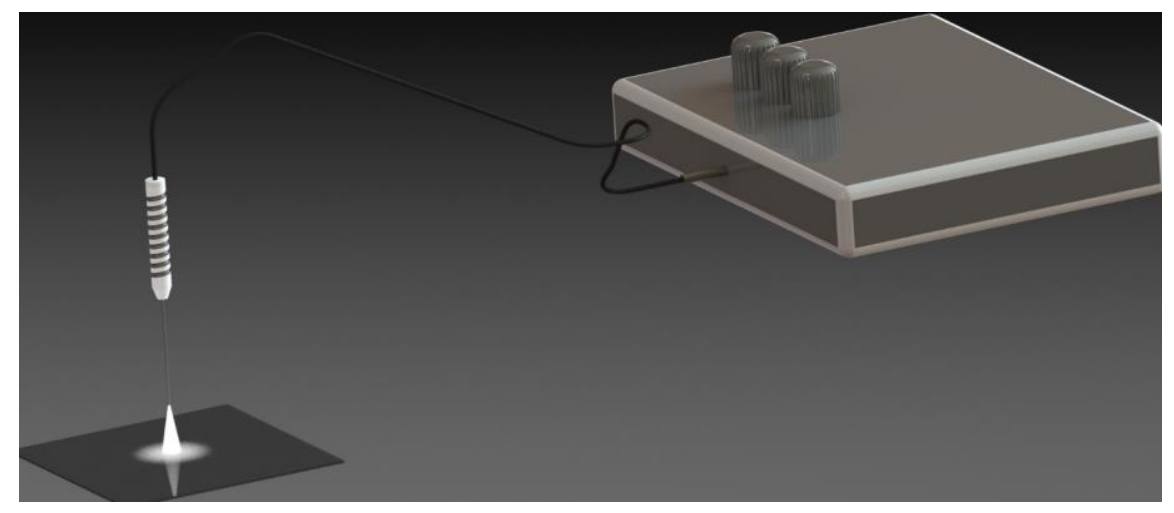

Рисунок 5. Инновационная надсистема комплексного лазерного инструмента для применения в офтальмологии 


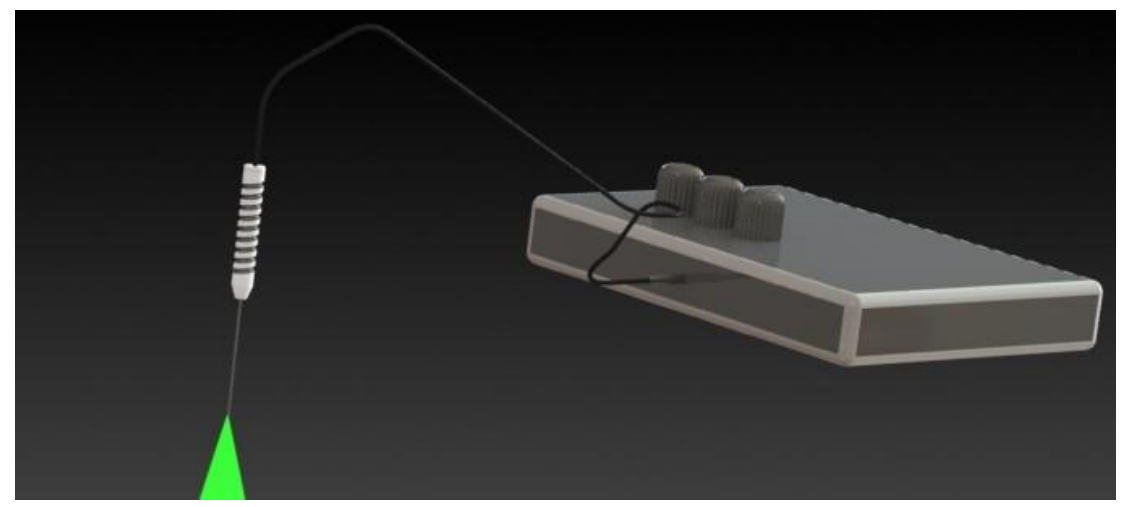

Рисунок 6. Инновационная надсистема комплексного лазерного инструмента для применения в офтальмологии

На рисунках 5 и 6 показана инновационная надсистема комплексного лазерного инструмента для применения в офтальмологии, работающая в режиме настройки и оптимизации параметров излучения. В системе одновременно работают три лазерных диода с различным цветом излучения, а также, как и в других примерах, используется одноразовый лазерный излучающий инструмент, причём этот инструмент, связан с указанными источниками лазерного излучения и их драйверами при помощи прецизионного оптического кабеля с инструментом на одном конце и с кодирующим устройством на другом конце, причём в системе в целом применяются люминофоры, задачей которых является получения требуемого спектра излучения одновременно с смягчением влияния лазерного излучения высокой концентрации и интенсивности на физиологические параметры организма пациента.

Для реализации комплексных решений в области специальных лазерных инструментов, одним из критичных факторов является ёмкость накопителей памяти и быстродействие всей цепочки - накопитель памяти - система управления и контроля исполнительный агрегат. Наличие значительных массивов информации требует также и необходимого уровня надёжности защитных технологий и их надёжной связи с накопителем памяти, в том числе и через элементы искусственного интеллекта и искусственные нейронные сети.

Для продолжения имеет смысл ознакомление с концептуальным решением по защитному кодированию, например оптического диска с инновационной многослойной структурой записи, увеличивающей ёмкость диска в тысячи раз. В качестве основного 
инструмента такой защитной системы выступает оптический диск, на котором нанесено кодирующее покрытие в кольцевой зоне, в которой в обычном диске нет информационной записи. В качестве вспомогательного инструмента выступает микросенсор, который встраивается в дисковод. Сигнал от микро-сенсора формируется при измерении толщины покрытия. Точность измерения - 100 ангстрем, и это величина, на которую отличается каждая группа дисков от другой группы. Сигнал от микро-сенсора является кодом для входа в массивы информации, размещённые в интернете.

Программное обеспечение должно давать возможность идентифицировать сигнал от микро-сенсора и в случае совпадения сигнала с эталонным открывать массивы информации и в процессе её скачивания продолжать контролировать достоверность сигнала до завершения процесса скачивания информации. Это даёт возможность предотвратить замену диска во время записи на нелицензированный. Подделать такой диск невозможно, так как толщина покрытия определяется при изготовлении, и, даже имея такой диск, невозможно им воспользоваться без микро-сенсора, настроенного на строго определённый характер сигнала. Диски и сенсоры могут выпускаться на любом сегодня существующем производстве оптических дисков; диски могут выпускаться сериями по 100 - 250 штук с одинаковой толщиной кодирующей ленты и с комплектом сенсоров. Каждый пользователь может приобрести одну или несколько серий дисков и использовать их при работе с интернетом. По такому же принципу программы и другая информация могут рассылаться пользователям, только в обратном порядке, что гарантирует полную конфиденциальность и защиту при нахождении в интернете от несанкционированных посланий и вирусов.

Это очень общая информация, и если её квалифицируют как заслуживающую внимания, то группа независимых изобретателей могла бы предельно точно детализировать этот проект. Ввиду того, что механическая часть этого проекта в принципе реализована, этот проект - это программное обеспечение, что может быть станет основой нового проекта в этом направлении также и с введением в проект элементов искусственного интеллекта и искусственных нейронных сетей. 